\title{
Multi-time correlations in relaxing quantum dynamical systems
}

\author{
Johan Andries ${ }^{1}$, Fabio Benatti ${ }^{2}$, Mieke De Cock ${ }^{3}$, Mark Fannes ${ }^{4}$ \\ Instituut voor Theoretische Fysica \\ Katholieke Universiteit Leuven \\ Celestijnenlaan 200D \\ B-3001 Heverlee, Belgium
}

\begin{abstract}
In this paper, we consider the long time asymptotics of multi-time correlation functions for quantum dynamical systems that are sufficiently random to relax to a "reference state". In particular, the evolution of such systems must have a continuous spectrum. Special attention is paid to general dynamical clustering conditions and their consequences for the structure of fluctuations of temporal averages.
\end{abstract}

\footnotetext{
1 Email: johan.andries@fys.kuleuven.ac.be

2 Permanent address: Dept. Theor. Phys. University of Trieste, Italy, Email: benatti@ts.infn.it

3 Onderzoeker FWO, Email: mieke.decock@fys.kuleuven.ac.be

4 Onderzoeksleider FWO, Email: mark.fannes@fys.kuleuven.ac.be
} 


\section{Introduction}

One of the main problems in quantum chaos is to understand the relaxation phenomena induced by the dynamics in systems with few degrees of freedom. Typically, the system relaxes on an appropriate time scale characteristic of the dynamics. Indeed, the spectrum of the evolution of chaotic quantum systems is usually discrete and observation of the system for very long times will reveal this discrete nature: the time correlation functions are quasiperiodic. The separation between the points of the spectrum depends on a quantization parameter such as $\hbar$ in the Chirikov kicked rotator [1] or on the dimension of an irreducible representation of $\mathrm{SU}(2)$ such as in the kicked top [2] or on that of an underlying Hilbert space as in the finite-dimensional Cat maps [3, 4]. When the quantization parameter tends to an appropriate limit, one obtains a classical dynamical system. We are not concerned with the statistical properties of eigenvalues and eigenvectors of such models, but rather with dynamical properties of expectations values. It is a typical feature that the classical limit and the limit for large times of such expectation values cannot be exchanged and so one can look for a joint limit obtained by a suitable scaling of the time with respect to the quantization parameter [5]. The aim is to extract by such a procedure true relaxation in rescaled time.

We don't address this scaling problem in this paper, but rather concentrate on the large time behaviour of model systems with already fully displayed relaxation as a consequence of basic dynamical randomness. Different types of behaviour are possible and we introduce a way of describing them by considering the asymptotic analysis of multi-time correlation functions [6]. In particular it appears that fluctuations around temporal averages are a useful tool to distinguish between various degrees of randomness. For instance, the distribution of fluctuations may be given by the usual Gaussian law, but also by the semicircle law or by more exotic laws. Such distributions have already been obtained considering fluctuations of observables obeying stochastic commutation relations [7]. In this paper, we show that deterministic dynamics, i.e. without any stochastic input, can lead to quite a variety of distributions for fluctuations. The appearance of unusual statistics, such as the free statistics associated with the semicircle law, is connected with chaotic features of the dynamics. By chaotic or random quantum features, we shall mean different degrees of clustering in time, namely different strengths with which events largely separated in time tend to become independent. Classically, one has the notion of mixing, whereas in quantum mechanics two notions are commonly considered, that of weak and strong clustering, the latter one implying the former. It turns out that the appearance of exotic statistics at the asymptotic level, rater than the common, Gaussian one, associated with the notion of classical independence and arising from the stronger clustering properties, is related to a finer distinction of possibilities between weak and strong clustering. We anyway expect to observe the emergence of such statistics, not only in dynamical systems with fully displayed relaxation, but also for fluctuations in appropriately scaled finite systems.

In Section 2, we review some notions of randomness for quantum dynamical systems. Section 3 deals with the construction of the asymptotics of multi-time correlation functions 
and provides hereby a useful setting for describing the law of large numbers for a sufficiently ergodic dynamical system. Then, we consider in Section 4 how a central limit theorem for fluctuations can be obtained. In Sections 5, we present the structures that arise when considering some simple examples of dynamical systems.

In this paper, we shall model quantum dynamical systems by triples $(\mathfrak{A}, \Theta, \phi)$ where - $\mathfrak{A}$ is a unital $\mathrm{C}^{*}$-algebra of observables

- $\Theta=\left\{\Theta_{t} \mid t \in \mathbf{R}\right.$ or $\left.\mathbf{Z}\right\}$ is a dynamical group of $*$-automorphisms of $\mathfrak{A}$ either in continuous or in discrete time and $X(t)$ will denote the observable $X \in \mathfrak{A}$ evolved up to time $t: X(t)=\Theta_{t}(X)$, and

- the reference state $\phi$ is assumed to be invariant under $\Theta$ i.e. $\phi \circ \Theta_{t}=\phi$.

General multi-time correlation functions are functions of the form

$$
\mathbf{t} \mapsto \phi\left(X^{(1)}\left(t_{\nu(1)}\right) X^{(2)}\left(t_{\nu(2)}\right) \cdots X^{(n)}\left(t_{\nu(n)}\right)\right)
$$

where the $X^{(j)}\left(t_{\nu(j)}\right)$ are operators at times $t_{\nu(j)}$ in $\mathfrak{A}, \mathbf{t}=\left\{t_{1}, t_{2}, \ldots\right\} \in \mathbf{Z}^{\mathbf{N}_{0}}$ and $\nu$ maps $\{1,2, \ldots, n\}$ into $\mathbf{N}_{0}$.

Usually, in quantum statistical mechanics, one considers time-ordered correlation functions. Since one expects observables largely separated in time to commute, such an ordering would be no restriction. We are interested, however, in a situation where a complex dynamics generates wildly fluctuating algebraic relations for observables largely separated in time. In such a case, commutation relations cannot be used to simplify correlation functions by grouping together observables at equal large times and, in order to obtain information about algebraic relations between observables largely separated in time, we have to consider general expressions as in (1). In particular, it is necessary to include the possibility of repeating a same time label within a correlation function. Then, the natural algebraic structure to consider is that of a countable free product $\mathfrak{A}_{\infty}$ of copies of $\mathfrak{A}$ [8], while the asymptotics of multi-time correlation functions will allow us to equip $\mathfrak{A}_{\infty}$ with an asymptotic state $\phi_{\infty}$. The probabilistic structure corresponding to such a state should reflect the essential features of the underlying dynamics.

We briefly remind the construction of $\mathfrak{A}_{\infty}$ as the free product $\star_{i \in \mathbf{N}_{0}} \mathfrak{A}_{i}$ and we shall refer to $\mathfrak{A}_{\infty}$ as the asymptotic free algebra associated with the dynamical system $(\mathfrak{A}, \Theta, \phi)$. Each of the algebras $\mathfrak{A}_{i}$ is a copy of the basic algebra $\mathfrak{A}$ of observables and $\mathfrak{A}_{\infty}$ is the universal C*-algebra generated by an identity element $\mathbb{I}$ and by "words" $w=X_{\nu(1)}^{(1)} X_{\nu(2)}^{(2)} \cdots X_{\nu(n)}^{(n)}$ that consist of concatenations of "letters" $X^{(j)} \in \mathfrak{A}$. The subscript $\nu(j)$ in $X_{\nu(j)}^{(j)}$ refers to which copy of $\mathfrak{A}$ the letter $X^{(j)}$ belongs to and any two consecutive subscripts are unequal. Concatenation, together with simplification rules, defines the product of words. More specifically, the rules for handling words are: for $X, Y \in \mathfrak{A}, \lambda \in \mathbf{C}, j \in \mathbf{N}_{0}$ and $w, w^{\prime}$ two generic words

$$
w \mathbb{I}_{j} w^{\prime} \Rightarrow w w^{\prime}
$$




$$
\begin{aligned}
w\left(X_{j}+\lambda Y_{j}\right) w^{\prime} & \Rightarrow w X_{j} w^{\prime}+\lambda w Y_{j} w^{\prime} \\
w X_{j} Y_{j} w^{\prime} & \Rightarrow w(X Y)_{j} w^{\prime} .
\end{aligned}
$$

Notice that the product $X Y$ in (2.c) is not concatenation, but rather the usual operator product in the algebra $\mathfrak{A}$. Moreover, the adjoint $w^{*}$ of a word $w=X_{\nu(1)}^{(1)} X_{\nu(2)}^{(2)} \cdots X_{\nu(n)}^{(n)}$ equals $\left(X^{(n) *}\right)_{\nu(n)}\left(X^{(n-1) *}\right)_{\nu(n-1)} \cdots\left(X^{(1) *}\right)_{\nu(1)}$.

\section{Random behaviours in quantum systems}

Before considering the problem of endowing $\mathfrak{A}_{\infty}$ with an asymptotic state, we present a hierarchy of ergodic properties typical for infinite quantum systems $[9,10]$. We shall formulate them for the case of discrete time dynamical systems $(\mathfrak{A}, \Theta, \phi)$.

Actually, our construction will be essentially based on the use of time averages of correlation functions of the form

$$
\overline{\phi(X Y(t) Z)}^{\text {av }}:=\lim _{T \rightarrow \infty} \frac{1}{T} \sum_{s=0}^{T-1} \phi(X Y(s) Z)
$$

where $X, Y$ and $Z$ are arbitrary observables in $\mathfrak{A}$.

If the dynamics of a system is sufficiently regular, observations turn out to be quite correlated, even when largely separated in time. We shall then associate various degrees of randomness with the strength of decorrelation properties of the dynamics, if any. The lowest degree of randomness is clustering in the mean

$$
\overline{\phi(X Y(t) Z)}^{\mathrm{av}}=\phi(X Z) \phi(Y), \quad X, Y, Z \in \mathfrak{A},
$$

next in the list comes weak clustering

$$
\lim _{t \rightarrow \infty} \phi(X Y(t) Z)=\phi(X Z) \phi(Y), \quad X, Y, Z \in \mathfrak{A}
$$

and we close the list with strong clustering

$$
\lim _{t \rightarrow \infty} \phi(X Y(t) Z S(t) T)=\phi(X Z T) \phi(Y S), \quad X, Y, Z, S, T \in \mathfrak{A}
$$

and hyper-clustering

$$
\lim _{\inf \left|t_{i}-t_{j}\right| \rightarrow \infty} \phi\left(X^{(1)}\left(t_{\nu(1)}\right) X^{(2)}\left(t_{\nu(2)}\right) \cdots X^{(n)}\left(t_{\nu(n)}\right)\right)=\prod_{j} \phi\left(\prod_{\kappa \in \nu^{-1}(j)} X^{(\kappa)}\right) .
$$

In formula (6.b), the limit is taken in such a way that all the times $t_{\nu(j)}$ and the differences between those with different indices go to infinity. Notice that, as in (1), repeated times 
are allowed in (6.b) and the arrow over the product at the right-hand side indicates that the order among operators at equal times has to be preserved since they do not commute in general. Among the previous properties, one can check that the following relations hold

$$
(6 . \mathrm{b}) \Leftrightarrow(6 . \mathrm{a}) \Rightarrow(5) \Rightarrow(4) \text {. }
$$

While the implications from left to right are evident, in order to deduce the equivalence in the first place, we use the notion of asymptotic Abelianess in time. Indeed, the different kinds of clustering (4), (5) and (6.a), imply the following degrees of asymptotic Abelianess: asymptotic Abelianess in the mean

$$
(4) \Longrightarrow \overline{\phi(S[X, Y(t)] Z)}^{\text {av }}=0 \quad \forall S, X, Y, Z \in \mathfrak{A},
$$

weak asymptotic Abelianess

$$
(5) \Longrightarrow \lim _{t \rightarrow \infty} \phi(S[X, Y(t)] Z)=0 \quad \forall S, X, Y, Z \in \mathfrak{A}
$$

and strong asymptotic Abelianess

$$
(6 . \mathrm{a}) \Longrightarrow \lim _{t \rightarrow \infty} \phi\left(S^{*}[X, Y(t)]^{*}[X, Y(t)] S\right)=0 \quad \forall S, X, Y \in \mathfrak{A} .
$$

Weak clustering plus strong asymptotic Abelianess imply strong clustering. Indeed

$$
\begin{aligned}
\lim _{t \rightarrow \infty} \phi(X Y(t) Z S(t) T) & =\lim _{t \rightarrow \infty} \phi(X Y(t) S(t) Z T)+\lim _{t \rightarrow \infty} \phi(X Y(t)[Z, S(t)] T) \\
& =\phi(X Z T) \phi(Y S) \quad \forall S, T, X, Y, Z \in \mathfrak{A} .
\end{aligned}
$$

The second limit tends to zero because of strong asymptotic Abelianess and the CauchySchwartz inequality

$$
\begin{aligned}
|\phi(X Y(t)[Z, S(t)] T)|^{2} & \leq \phi\left(X Y(t) Y(t)^{*} X^{*}\right) \phi\left(T^{*}[Z, S(t)]^{*}[Z, S(t)] T\right) \\
& \leq\|X\|^{2}\|Y\|^{2} \phi\left(T^{*}[Z, S(t)]^{*}[Z, S(t)] T\right)
\end{aligned}
$$

while the first one gives the result because of weak-clustering. By a similar argument, (6.a) implies (6.b) and therefore the equivalence in (7) holds. We just sketch here the main idea of the argument by considering the case of three equal times. In a first step, for fixed $X, Y, Q, S, T, U$ and $Z$ in $\mathfrak{A}$, write

$$
\begin{gathered}
\phi(X Y(t) S Z(t) T U(t) Q)=\phi(X S Y(t) Z(t) T U(t) Q)+ \\
\phi(X[Y(t), S] Z(t) T U(t) Q) .
\end{gathered}
$$

By the Cauchy-Schwarz inequality and $\phi\left(a b b^{*} a^{*}\right) \leq\|b\|^{2} \phi\left(a a^{*}\right)$ we obtain

$$
|\phi(X[Y(t), S] Z(t) T U(t) Q)|^{2} \leq\|Z\|^{2}\|T\|^{2}\|U\|^{2}\|Q\|^{2} \phi\left(X[Y(t), S][Y(t), S]^{*} X^{*}\right) .
$$

Assuming strong asymptotic Abelianess (8.c), the second term in (9) vanishes when $t$ tends to infinity. Repeating the same procedure on the first term, we collect together $(Y Z U)(t)$ and the conclusion follows from weak-clustering. Indeed, the condition that inf $\left|t_{i}-t_{j}\right| \rightarrow \infty$ means that all different times are so largely separated that the regrouped correlation functions cluster asymptotically with respect to any of them. 


\section{The asymptotic multi-time correlations}

Consider, as in (1), a multi-time correlation function

$$
\mathbf{t} \mapsto \phi\left(X^{(1)}\left(t_{\nu(1)}\right) X^{(2)}\left(t_{\nu(2)}\right) \cdots X^{(n)}\left(t_{\nu(n)}\right)\right) .
$$

We may restrict the map $\nu:\{1,2, \ldots, n\} \rightarrow \mathbf{N}_{0}$ in such a way that if a $\nu(j)$ appears somewhere in the operator product in (10), then all smaller natural numbers must have already appeared at least once to the left of $\nu(j)$. More formally

$$
\text { if } 1 \leq \ell<\nu(j) \text {, then there exists } 1 \leq i<j \text { such that } \nu(i)=\ell \text {. }
$$

As a consequence of this prescription, $\nu(\{1,2, \ldots, n\})=\{1,2, \ldots, k\}$ with $k \leq n$ due to possible repetitions of a label. Notice that this is a convenient way of rewriting the multi-time correlation functions that were introduced in (1): e.g. $\mathbf{t} \mapsto \phi\left(X\left(t_{3}\right) Y\left(t_{2}\right)\right)$ is not excluded by the rule of above for $\nu$ since it equals $\left.\mathbf{t} \mapsto \phi\left(\mathbb{I}\left(t_{1}\right) \mathbb{I}\left(t_{2}\right)\right) X\left(t_{3}\right) Y\left(t_{2}\right)\right)$. The reason for making this specific choice in writing correlation functions is to have all labels $1,2, \ldots, n$ appear, in increasing order and possibly with repetitions, in a correlation function if the label $n$ appears. This will become useful later on when we consider time averages. The multi-time correlation functions (10) form a self-adjoint linear space containing the constant function.

We shall describe the asymptotics of multi-time correlation functions in terms of a state $\phi_{\infty}$ on the asymptotic free algebra $\mathfrak{A}_{\infty} . \phi_{\infty}$ is obtained by averaging multi-time correlation functions. We now generalize the procedure of averaging considered in (3) for a single-time correlation function and briefly recall the notion of invariant mean [11]. A mean Avg on a set $X$ is a normalized and positive linear functional on a self-adjoint linear space $\mathfrak{X}$ of bounded complex-valued functions on $X$ containing the constant function and closed for the \|\|$_{\infty}$-norm. If $\mathfrak{G}$ is a group of transformations of $X$, leaving $\mathfrak{X}$ globally invariant, then Avg is called invariant if $\operatorname{Avg}(f)=\operatorname{Avg}(f \circ \gamma)$.

As we consider discrete time dynamical systems $(\mathfrak{A}, \Theta, \phi)$, we take $\mathbf{Z}^{\mathbf{N}_{0}}$ for the space $X$. The group $\mathfrak{G}$ will consist of multi-time translations on $X$ given by

$$
\gamma_{\mathbf{s}}: X \rightarrow X: \mathbf{t} \mapsto \mathbf{t}-\mathbf{s}, \quad \mathbf{s}=\left\{s_{1}, s_{2}, \ldots\right\} \in \mathbf{Z}^{\mathbf{N}_{0}} .
$$

The function space $\mathfrak{X}$ on which we shall consider means is the closure of the linear space of multi-time correlation functions. $\mathfrak{X}$ is invariant under the group action $f \mapsto f \circ \gamma_{\mathbf{s}}$ since $\Theta$ is an automorphism of $\mathfrak{A}$.

In general, we want to explore the possibility of defining a positive, normalized linear functional (a state) $\phi_{\infty}$ on the linear span of the words in the asymptotic free algebra, via some mean "Avg" defined on the space of multi-time correlation functions. 
We proceed as follows: first we rewrite a general word $w=X_{\mu(1)}^{(1)} X_{\mu(2)}^{(2)} \cdots X_{\mu(n)}^{(n)}$ without restrictions on the map $\mu$ except that consecutive subindices $\mu(j)$ and $\mu(j+1)$ are different. By inserting an appropriate number of identities we can write in a unique way $w=Y_{\nu(1)}^{(1)} Y_{\nu(2)}^{(2)} \cdots Y_{\nu(r)}^{(r)}$, where the $Y$ are either identities or are $X$ 's and where $\nu$ satisfies (11). E.g. $w=X_{2}$ is rewritten as $w=\mathbb{I}_{1} X_{2}$ and $w=X_{2} Y_{1} Z_{2} W_{4}$ becomes $w=\mathbb{I}_{1} X_{2} Y_{1} Z_{2} \mathbb{I}_{3} W_{4}$. Given the space of multi-time correlation functions of a dynamical system $(\mathfrak{A}, \Theta, \phi)$, we shall consider, when existing, the linear functionals

$$
\phi_{\infty}\left(X_{\nu(1)}^{(1)} X_{\nu(2)}^{(2)} \cdots X_{\nu(n)}^{(n)}\right):=\operatorname{Avg}\left(\mathbf{t} \mapsto \phi\left(X^{(1)}\left(t_{\nu(1)}\right) X^{(2)}\left(t_{\nu(2)}\right) \cdots X^{(n)}\left(t_{\nu(n)}\right)\right)\right)
$$

where $\nu$ maps $\{1,2, \ldots, n\}$ into $\{1,2, \ldots, s\}$. Furthermore, we restrict ourselves to those means Avg on $\mathfrak{X}$ that satisfy the following strong compatibility condition:

if $\left(t_{1}, t_{2}, \ldots, t_{n}\right) \mapsto f\left(t_{1}, t_{2}, \ldots, t_{n}\right)$ is a uniform limit of multi-time correlation functions, and if $f$ depends only on a subset $\left\{t_{i(1)}, t_{i(2)}, \ldots, t_{i(k)}\right\}$ of variables where $i:\{1,2, \ldots, k\} \rightarrow\{1,2, \ldots, n\}$ is an order preserving injection, that is $f\left(t_{1}, t_{2}, \ldots, t_{n}\right)=g\left(t_{i(1)}, t_{i(2)}, \ldots, t_{i(k)}\right)$, then $\boldsymbol{A v g}(f)=\operatorname{Avg}(g)$.

Proposition 1. If Avg exists as an invariant mean on the space of multi-time correlation functions and satisfies the strong compatibility condition (13), then the functional $\phi_{\infty}$ defined in (12) extends to a state on $\mathfrak{A}_{\infty}$. Furthermore,

$$
\phi_{\infty} \circ \boldsymbol{\Theta}_{\mathbf{s}}=\phi_{\infty} \quad \text { and } \quad \phi_{\infty} \circ \alpha_{\theta}=\phi_{\infty}
$$

where $\boldsymbol{\Theta}_{\mathbf{s}}$ satisfies $\boldsymbol{\Theta}_{\mathbf{s}}\left(X_{j}\right):=\left(\Theta_{s_{j}}(X)\right)_{j}$ for $j \in \mathbf{N}_{0}$ and $X \in \mathfrak{A}$, whereas $\theta$ is any order preserving injective transformation of $\mathbf{N}_{0}$ and $\alpha_{\theta}$ is the $*$-homomorphism of $\mathfrak{A}_{\infty}$ determined by $\alpha_{\theta}\left(X_{j}\right):=X_{\theta(j)}$.

Proof: Normalization and linearity are a consequence of (2.a) and (2.b). In order to prove positivity we have to check that for any finite linear combination $W=\sum_{w} \lambda_{w} w$ of words one has $\phi_{\infty}\left(W^{*} W\right) \geq 0$. From definition (12) it turns out that $\phi_{\infty}\left(W^{*} W\right)$ is the multiple average of the expectation of a positive operator in the state $\phi$, hence positive. Multi-time invariance follows from the invariance of the mean. Again, instead of producing a formal proof of the invariance of $\phi_{\infty}$ under $\alpha_{\theta}$, we present a simple example which clarifies the essential mechanism. We show that $\phi_{\infty}\left(X_{2} Y_{1} Z_{2}\right)=\phi_{\infty}\left(X_{3} Y_{1} Z_{3}\right)$, in which case $\theta(1)=1$ and $\theta(2)=3$. Relabelling dummy variables and using condition (13), we obtain

$$
\begin{aligned}
\phi_{\infty}\left(X_{3} Y_{1} Z_{3}\right) & =\operatorname{Avg}\left(\left(t_{1}, t_{2}, t_{3}, \ldots\right) \mapsto \phi\left(\mathbb{I}\left(t_{1}\right) \mathbb{I}\left(t_{2}\right) X\left(t_{3}\right) Y\left(t_{1}\right) Z\left(t_{3}\right)\right)\right) \\
& =\operatorname{Avg}\left(\left(t_{1}, t_{2}, t_{3}, \ldots\right) \mapsto \phi\left(\mathbb{I}\left(t_{1}\right) X\left(t_{2}\right) Y\left(t_{1}\right) Z\left(t_{2}\right)\right)\right) \\
& =\phi_{\infty}\left(X_{2} Y_{1} Z_{2}\right) .
\end{aligned}
$$


In the following, we shall fix a definite averaging procedure and construct asymptotic states $\phi_{\infty}$ averaging over the different times in successive order

$$
\begin{aligned}
\operatorname{Avg}\left(\mathbf{t} \mapsto f\left(t_{1}, t_{2}, \ldots, t_{n}\right)\right):= \\
\quad \operatorname{Avg}\left(t_{n} \mapsto \cdots \operatorname{Avg}\left(t_{2} \mapsto \operatorname{Avg}\left(t_{1} \mapsto f\left(t_{1}, t_{2}, \ldots, t_{n}\right)\right)\right) \cdots\right)
\end{aligned}
$$

More general procedures are possible by considering coupled limits: e.g. one could try to construct a mean by averaging functions $\left(t_{1}, t_{2}, \ldots, t_{n}\right) \mapsto f\left(t_{1}, t_{2}, \ldots, t_{n}\right)$ of $n$ arguments over $n$-dimensional cubes

$$
\operatorname{Avg}\left(\left(t_{1}, t_{2}, \ldots, t_{n}\right) \mapsto f\left(t_{1}, t_{2}, \ldots, t_{n}\right)\right):=\lim _{L \rightarrow \infty} \frac{1}{L^{n}} \sum_{t_{1}=1}^{L} \cdots \sum_{t_{n}=1}^{L} f\left(t_{1}, \ldots, t_{n}\right)
$$

Such a mean is also strongly compatible. Notice, however, that, choosing different singletime averages Avg in (14), we can in general violate the strong compatibility condition (13). Here follows an example with two times and two different single-time averages

$$
\begin{aligned}
\operatorname{Avg}_{2}\left(t_{2} \mapsto \operatorname{Avg}_{1}\left(t_{1} \mapsto f\left(t_{2}\right)\right)\right) & =\operatorname{Avg}_{2}(t \mapsto f(t)) \\
& \neq \operatorname{Avg}_{1}(t \mapsto f(t)) \\
& =\operatorname{Avg}_{2}\left(t_{2} \mapsto \operatorname{Avg}_{1}\left(t_{1} \mapsto f\left(t_{1}\right)\right)\right)
\end{aligned}
$$

Quite to the other extreme, there are examples of dynamical systems and of averages of their multi-time correlation functions that are not only strongly compatible but even permutation invariant in the sense that

$$
\operatorname{Avg}\left(\alpha_{\pi}(f)\right)=\operatorname{Avg}(f)
$$

for any local permutation $\pi$ of the natural numbers, that is for any bijection $\pi$ from $\mathbf{N}_{0}$ into $\mathbf{N}_{0}$ that leaves all elements invariant except for a finite number of them. $\alpha_{\pi}$ acts on a correlation function $\mathbf{t} \mapsto f\left(t_{1}, t_{2}, \ldots\right)$ as $\alpha_{\pi}(f)\left(t_{1}, t_{2}, \ldots\right)=f\left(t_{\pi(1)}, t_{\pi(2)}, \ldots\right)$. As a consequence of (15), the asymptotic states $\phi_{\infty}$ defined by permutation invariant multi-time averages will also be permutation invariant on the asymptotic algebra $\mathfrak{A}_{\infty}$, namely

$$
\phi_{\infty}\left(X_{\nu(1)}^{(1)} X_{\nu(2)}^{(2)} \cdots X_{\nu(n)}^{(n)}\right)=\phi_{\infty}\left(X_{\pi \circ \nu(1)}^{(1)} X_{\pi \circ \nu(2)}^{(2)} \cdots X_{\pi \circ \nu(n)}^{(n)}\right) \quad \forall X^{(j)} \in \mathfrak{A} .
$$

The asymptotic states $\phi_{\infty}$ are always permutation invariant when the basic dynamical system $(\mathfrak{A}, \Theta, \phi)$ is strongly clustering. In order to prove this, we introduce a useful technical result. 
Lemma 1. For $d, k \in \mathbf{N}$ define

$$
\Delta_{d}^{k}\left(t_{1}, \ldots, t_{k}\right)= \begin{cases}0 & \text { if }\left|t_{i}-t_{j}\right| \leq d \text { for some } 1 \leq i \neq j \leq k \\ 1 & \text { else }\end{cases}
$$

Then, if the multiple average (14) of a uniformly bounded function $f: \mathbf{Z}^{k} \rightarrow \mathbf{C}$ exists, we have

$$
\begin{aligned}
& \operatorname{Avg}\left(t_{k} \mapsto \cdots \operatorname{Avg}\left(t_{2} \mapsto \operatorname{Avg}\left(t_{1} \mapsto f\left(t_{1}, t_{2}, \ldots, t_{k}\right)\right)\right) \cdots\right)= \\
& \operatorname{Avg}\left(t_{k} \mapsto \cdots \operatorname{Avg}\left(t_{2} \mapsto \operatorname{Avg}\left(t_{1} \mapsto \Delta_{d}^{k}\left(t_{1}, \ldots, t_{k}\right) f\left(t_{1}, t_{2}, \ldots, t_{k}\right)\right)\right) \cdots\right)
\end{aligned}
$$

Proof: For fixed $d$, choose $t_{1}, \ldots, t_{i-1}, t_{i+1}, \ldots, t_{k} \in \mathbf{Z}$ in such a way that the function $\Delta_{d}^{k-1}\left(t_{1}, \ldots, t_{i-1}, t_{i+1}, \ldots, t_{k}\right)=1$. Then, $\Delta_{d}^{k}\left(t_{1}, \ldots, t_{i}, \ldots, t_{k}\right)=0$ for only a finite number of values of $t_{i} \in \mathbf{Z}$ which implies that

$$
\begin{aligned}
\operatorname{Avg}\left(t_{i} \mapsto \Delta_{d}^{k}\right. & \left.\left(t_{1}, \ldots, t_{i}, \ldots, t_{k}\right) g\left(t_{1}, \ldots, t_{i}, \ldots, t_{k}\right)\right) \\
& =\Delta_{d}^{k-1}\left(t_{1}, \ldots, t_{i-1}, t_{i+1}, \ldots, t_{k}\right) \operatorname{Avg}\left(t_{i} \mapsto g\left(t_{1}, \ldots, t_{i}, \ldots, t_{k}\right)\right)
\end{aligned}
$$

for a uniformly bounded $g: \mathbf{Z}^{k} \rightarrow \mathbf{C}$. By successively applying this observation to

$$
\operatorname{Avg}\left(t_{n} \mapsto \cdots \operatorname{Avg}\left(t_{2} \mapsto \operatorname{Avg}\left(t_{1} \mapsto \Delta_{d}^{n}\left(t_{1}, \ldots, t_{n}\right) f\left(t_{1}, t_{2}, \ldots, t_{n}\right)\right)\right) \cdots\right)
$$

the lemma follows. Whereas in the statement of the lemma a definite order of the time averages has been specified, its proof is independent of it.

Proposition 2. Let $(\mathfrak{A}, \Theta, \phi)$ be strongly clustering as in (6.a), equivalently (6.b). Then, if the multiple average in (14) exists, it defines a permutation invariant asymptotic state $\phi_{\infty}$ on $\mathfrak{A}_{\infty}$.

Proof: Let us consider a multi-time correlation function

$$
\phi\left(X^{(1)}\left(t_{\nu(1)}\right) X^{(2)}\left(t_{\nu(2)}\right) \cdots X^{(n)}\left(t_{\nu(n)}\right)\right)
$$

where, because of strong compatibility, we can assume that $\nu:\{1,2, \ldots, n\} \rightarrow\{1,2, \ldots, k\}$ with $k \leq n$. Thus, we collect all $i \in\{1,2, \ldots, n\}$ such that $\nu(i)=p$ into subsets $I_{p}$. Because of strong-clustering, and thus of hyper-clustering (6.b), for any $\epsilon>0$, we can choose $d>0$ such that $\left|t_{i}-t_{j}\right| \geq d$ for all $t_{i} \neq t_{j}$, with $i, j \in\{1,2, \ldots, k\}$, implies

$$
\left|\phi\left(X^{(1)}\left(t_{\nu(1)}\right) X^{(2)}\left(t_{\nu(2)}\right) \cdots X^{(n)}\left(t_{\nu(n)}\right)\right)-\prod_{p=1}^{k} \phi\left(\prod_{i \in I_{p}} X^{(i)}\right)\right| \leq \epsilon .
$$


Thus, when evaluating $\phi_{\infty}\left(X_{\nu(1)}^{(1)} X_{\nu(2)}^{(2)} \cdots X_{\nu(n)}^{(n)}\right)$ via the prescription (14), we can use Lemma 1 and a function $\Delta_{d}^{k}\left(t_{1}, t_{2}, \ldots, t_{k}\right)$ to estimate

$$
\left|\phi_{\infty}\left(X_{\nu(1)}^{(1)} X_{\nu(2)}^{(2)} \cdots X_{\nu(n)}^{(n)}\right)-\prod_{p=1}^{k} \phi\left(\vec{\prod}_{i \in I_{p}} X^{(i)}\right)\right| \leq \epsilon
$$

Therefore, in the case of strong clustering, in whichever order the single time averages are performed, the multiple average (14) agrees with the time limit (6.b) of multi-time correlation functions. The ensuing asymptotic state $\phi_{\infty}$ is thus permutation invariant. Moreover, from time invariance of $\phi$, we have that $\phi_{\infty}\left(X_{j}\right)=\phi(X)$ for any $j \in \mathbf{N}_{0}$ and $X \in \mathfrak{A}$. Therefore, with $i \in I_{p}$ implying $\nu(i)=p$,

$$
\phi_{\infty}\left(X_{\nu(1)}^{(1)} X_{\nu(2)}^{(2)} \cdots X_{\nu(n)}^{(n)}\right)=\prod_{p=1}^{k} \phi_{\infty}\left(\vec{\prod}_{i \in I_{p}} X^{(i)}\right)
$$

When the basic dynamics is strongly clustering, the structure of the expectations on $\mathfrak{A}_{\infty}$ calculated with respect to the asymptotic state $\phi_{\infty}$ corresponds to the usual notion of commutative independence of random variables. Indeed, $\phi_{\infty}$ vanishes on the two-sided ideal of $\mathfrak{A}_{\infty}$ generated by commutators of letters sitting in different copies of $\mathfrak{A}$ in $\mathfrak{A}_{\infty}$, i.e. by $\left\{\left[X_{j}, Y_{k}\right] \mid j \neq k, X, Y \in \mathfrak{A}\right\}$. We may therefore think of $\phi_{\infty}$ as an infinite product state on the minimal tensor product of a countable number of copies of $\mathfrak{A}$.

A very different notion of independence, called freeness or free independence, has been recently introduced in the realm of non-commutative probability [8]. This notion corresponds to the following structure for the correlation functions of a state $\psi$ on a free product $\star_{j} \mathfrak{B}_{j}$ of $\mathrm{C}^{*}$-algebras $\mathfrak{B}_{j}$

$$
\psi\left(X_{j_{1}}^{(1)} X_{j_{2}}^{(2)} \cdots X_{j_{n}}^{(n)}\right)=0
$$

whenever $\psi\left(X_{j_{k}}^{(k)}\right)=0$ and $j_{k} \neq j_{k+1}$ for all $k$. Accordingly, one refers to $\psi$ as to the free product of the states $\psi_{j}$, where $\psi_{j}$ is the restriction of $\psi$ to $\mathfrak{B}_{j}$.

Freeness is totally incompatible with statistical independence as in (17). In fact, if $\phi_{\infty}$ satisfied both freeness and commutative independence, then we would have for any centred observable $X$, i.e. $\phi(X)=0$, that

$$
0=\phi_{\infty}\left(X_{0}^{*} X_{1}^{*} X_{0} X_{1}\right)=\phi\left(X^{*} X\right)^{2}
$$




\section{Temporal fluctuations}

The two cases of commutative and free independence, presented in the previous section, are somehow extreme. Many other possibilities for the structure of $\phi_{\infty}$ may arise. In general, we cannot hope to obtain a comprehensive description of these structures. A more manageable framework is provided by "fluctuations". They are natural objects to consider as we may think of the state $\phi_{\infty}$ as determining the Law of Large Numbers for the dynamical system $(\mathfrak{A}, \Theta, \phi)$. Fluctuations are thus on the level of the Central Limit Theorem.

Definition. Let $N \in \mathbf{N}_{0}$ and $X \in \mathfrak{A}$. A local fluctuation $F_{N}(X)$ is the following element of $\mathfrak{A}_{\infty}$

$$
F_{N}(X):=\frac{1}{\sqrt{N}} \sum_{i=1}^{N}\left(X_{i}-\phi(X) \mathbb{I}\right)
$$

It is our aim to discuss the limiting behaviour of $F_{N}(X)$ when $N$ tends to infinity, namely to study limits of correlations such as

$$
\lim _{N \rightarrow \infty} \phi_{\infty}\left(F_{N}\left(X^{(1)}\right) F_{N}\left(X^{(2)}\right) \cdots F_{N}\left(X^{(r)}\right)\right)
$$

and to establish an algebraic central limit theorem.

In a stronger sense, one may try to reconstruct possible algebraic relations of global fluctuations $F(X)$, if any, by means of the functional $\Phi$ on global fluctuations defined by

$$
\Phi\left(F\left(X^{(1)}\right) F\left(X^{(2)}\right) \cdots F\left(X^{(r)}\right)\right):=\lim _{N \rightarrow \infty} \phi_{\infty}\left(F_{N}\left(X^{(1)}\right) F_{N}\left(X^{(2)}\right) \cdots F_{N}\left(X^{(r)}\right)\right) .
$$

Notice that, in the case of commutative, respectively free independence, the linear functional $\Phi$ on the r.h.s. of (19) is in fact a well-defined state on the algebra of fluctuations $[12,7]$ such that they become Gaussianly, respectively semicircularly, distributed, non-commutative, random variables.

We shall restrict our considerations to averages of multi-time correlation functions of the form (14) and study in some generality the limit (18) by adapting an argument in [13]. We show that a clustering condition stronger than weak clustering (5), but weaker than strong clustering (6.a), is sufficient to ensure that only moments of even order contribute to the limit joint distribution of fluctuations.

Proposition 3. Let us assume that the quantum dynamical system $(\mathfrak{A}, \Theta, \phi)$ satisfies the following cluster condition

$$
\lim _{\inf \left|t_{i}-t_{j}\right| \rightarrow \infty} \phi\left(Z^{(1)}\left(t_{\nu(1)}\right) \cdots Z^{(j)}\left(t_{\nu(j)}\right) Y Z^{(j+1)}\left(t_{\nu(j+1)}\right) \cdots Z^{(n)}\left(t_{\nu(n)}\right)\right)=0
$$


whenever $Y$ is centred and $\nu$ maps $\{1,2, \ldots, n\}$ into $\mathbf{N}_{0}$, where, as in (6.b), $\lim _{\mathrm{inf}}\left|t_{i}-t_{j}\right| \rightarrow \infty$ means that all times and differences of different times go to infinity. If the multiple average (14) exists, the state $\phi_{\infty}$ it defines on the asymptotic algebra $\mathfrak{A}_{\infty}$ is such that, with $X^{(1)}, \ldots, X^{(r)}$ in $\mathfrak{A}$ centred observables,

$$
\lim _{N \rightarrow \infty} \phi_{\infty}\left(F_{N}\left(X^{(1)}\right) F_{N}\left(X^{(2)}\right) \cdots F_{N}\left(X^{(r)}\right)\right)= \begin{cases}0 & r=2 n+1 \\ \frac{1}{n !} \sum_{\nu}^{(2)} \phi_{\infty}\left(X_{\nu(1)}^{(1)} \cdots X_{\nu(2 n)}^{(2 n)}\right) & r=2 n .\end{cases}
$$

$\sum_{\nu}^{(2)}$ means that we have to sum over all partitions $\nu$ of $\{1,2, \ldots, 2 n\}$ into pairs $\left(\left(\alpha_{1}, \beta_{1}\right)\right.$, $\left.\left(\alpha_{2}, \beta_{2}\right), \ldots,\left(\alpha_{n}, \beta_{n}\right)\right)$ i.e. we choose sites $\alpha_{j}<\beta_{j}$ such that $\nu\left(\alpha_{j}\right)=\nu\left(\beta_{j}\right)=j$ with $j$ running from 1 to $n$.

Proof: We have to compute the limit for large $N$ of

$$
\phi_{\infty}\left(F_{N}\left(X^{(1)}\right) F_{N}\left(X^{(2)}\right) \cdots F_{N}\left(X^{(r)}\right)\right)=\frac{1}{N^{r / 2}} \sum_{\mathbf{k} \in\{1, \ldots, N\}^{r}} \phi_{\infty}\left(X_{k_{1}}^{(1)} \cdots X_{k_{r}}^{(r)}\right)
$$

where $\mathbf{k}=\left\{k_{1}, \ldots, k_{r}\right\}$. We first concentrate on the contributions to (21) where at least one of the indices $k_{1}, k_{2}, \ldots, k_{r}$ appears only once and show that all of them vanish. Let $k_{p}$ be such an index. Due to strong compatibility, we may always assume that the map $i \mapsto k_{i}$ transform $\{1,2, \ldots, r\}$ into $\{1,2, \ldots, s\}$, with $s \leq r$ due to possible repetitions of an index $k_{i}$. However, by hypothesis, the index $k_{p}$ appears just once, meaning that the time $t_{k_{p}}$ does not explicitly appear in the words

$X^{(1)}\left(t_{k_{1}}\right) X^{(2)}\left(t_{k_{2}}\right) \cdots X^{(p-1)}\left(t_{k_{p-1}}\right) \quad$ and $\quad X^{(p+1)}\left(t_{k_{p+1}}\right) X^{(p+2)}\left(t_{k_{p+2}}\right) \cdots X^{(r)}\left(t_{k_{r}}\right)$.

Let $X^{(p)}$ be a centred observable and use that $\phi_{\infty}$ is defined by (14) and that the dynamical system $(\mathfrak{A}, \Theta, \phi)$ satisfies condition (20). Then, Lemma 1 guarantees that, given any $\epsilon>0$, we can find a corridor function $\Delta_{d}^{s}\left(t_{1}, t_{2}, \ldots, t_{s}\right)$ with $d$ so large that

$$
\begin{aligned}
& \left|\phi_{\infty}\left(X_{k_{1}}^{(1)} \cdots X_{k_{p-1}}^{(p-1)} X_{k_{p}}^{(p)} X_{k_{p+1}}^{(p+1)} \cdots X_{k_{r}}^{(r)}\right)\right|= \\
& \mid \operatorname{Avg}\left(t _ { s } \mapsto \cdots \operatorname { A v g } \left(t _ { 2 } \mapsto \operatorname { A v g } \left(t_{1} \mapsto\right.\right.\right. \\
& \left.\left.\left.\quad \phi\left(X^{(1)}\left(t_{k_{1}}\right) \cdots X^{(p-1)}\left(t_{k_{p-1}}\right) X^{(p)}\left(t_{k_{p}}\right) X^{(p+1)}\left(t_{k_{p+1}}\right) \cdots X^{(r)}\left(t_{k_{r}}\right)\right)\right)\right) \cdots\right) \mid \leq \epsilon .
\end{aligned}
$$

Therefore, the only non-zero contributions come from terms where none of the subindices $k_{j}$ appears alone.

Next, we prove that, in the limit of large $N$, all those contributions vanish which come from terms where all indices appear twice and at least one thrice. As $\phi_{\infty}$ is a state, we have the a priori estimate

$$
\left|\phi_{\infty}\left(X_{k_{1}}^{(1)} \cdots X_{k_{r}}^{(r)}\right)\right| \leq \prod_{\ell=1}^{r}\left\|X^{(\ell)}\right\|
$$


Let us partition $\{1,2, \ldots, r\}$ into $s$ groups of equal indices and let $r_{1}, r_{2}, \ldots, r_{s}$ denote the number of elements in each such group. By assumption, $r_{j} \geq 2$, at least one of the $r_{j} \geq 3$ and $r_{1}+r_{2}+\cdots+r_{s}=r>2 s$. The contribution of all these terms can be estimated as follows: any partition $\mathbf{k}:\{1,2, \ldots, r\} \mapsto\left\{k_{1}, k_{2}, \ldots, k_{s}\right\}$, where $k_{j} \in\{1,2, \ldots, N\}$, can be written as $\mathbf{k}=\theta \circ \nu$, with $\nu:\{1,2, \ldots, r\} \mapsto\{1,2, \ldots, s\}$ a partition and $\theta$ : $\{1,2, \ldots, s\} \mapsto\{1,2, \ldots, N\}$ an order preserving injection. There are $\left(\begin{array}{c}N \\ s\end{array}\right)$ of such order preserving injections. Thus, the contribution of the type considered is bounded from above by

$$
\frac{1}{N^{r / 2}} \sum_{s<\frac{r}{2}} A_{s}\left(\begin{array}{c}
N \\
s
\end{array}\right) \prod_{\ell=1}^{r}\left\|X^{(\ell)}\right\| .
$$

$A_{s}$ is the number of partitions $\nu:\{1,2, \ldots, r\} \mapsto\{1,2, \ldots, s\}$ such that each $1<j<s$ appears at least twice. Such an upper bound tends to zero when $N$ tends to infinity.

Therefore, we remain with contributions of the form $\phi_{\infty}\left(X_{k_{1}}^{(1)} \cdots X_{k_{r}}^{(r)}\right)$ where the $k_{j}$ run from 1 to $N$ and each of them appears exactly twice, whence $r$ has to be even, say $r=$ $2 n$. These contributions are given by $\phi_{\infty}\left(X_{\nu(1)}^{(1)} \cdots X_{\nu(2 n)}^{(2 n)}\right)$, where $\nu:\{1, \ldots, 2 n\} \mapsto$ $\{1, \ldots, n\}$ is a (not necessarily ordered) pair partition, that is, for any $j \in\{1,2, \ldots, n\}$, there exist two, and only two, indices $\left(\alpha_{j}, \beta_{j}\right) \in\{1,2, \ldots, 2 n\}$ such that $\nu\left(\alpha_{j}\right)=\nu\left(\beta_{j}\right)=$ $j$. In fact, as before: any pair partition $\mathbf{k}:\{1,2, \ldots, 2 n\} \mapsto\left\{k_{1}, k_{2}, \ldots, k_{n}\right\}$, where $k_{j} \in\{1,2, \ldots, N\}$, can be written as $\mathbf{k}=\theta \circ \nu$ with $\nu:\{1,2, \ldots, 2 n\} \mapsto\{1,2, \ldots, n\}$ a pair partition and $\theta:\{1,2, \ldots, n\} \mapsto\{1,2, \ldots, N\}$ an order preserving injection. There being $\left(\begin{array}{l}N \\ n\end{array}\right)$ of such injections, the sum of contributions from generic pair partitions $\mathbf{k}$ : $\{1,2, \ldots, 2 n\} \mapsto\{1,2, \ldots, N\}$ can be simplified to

$$
\sum_{\mathbf{k}} \phi_{\infty}\left(X_{k_{1}}^{(1)} X_{k_{2}}^{(2)} \cdots X_{k_{r}}^{(r)}\right)=\left(\begin{array}{c}
N \\
n
\end{array}\right) \sum_{\nu}^{(2)} \phi_{\infty}\left(X_{\nu(1)}^{(1)} X_{\nu(2)}^{(2)} \cdots X_{\nu(n)}^{(2 n)}\right)
$$

where $\nu$ is any pair partition of $\{1,2, \ldots, 2 n\} \mapsto\{1,2, \ldots, n\}$. As $\lim _{N} N^{-n}\left(\begin{array}{c}N \\ n\end{array}\right)=1 / n$ !, the result follows.

\section{Remarks}

a. Condition (20) is only sufficient to obtain the central limit theorem of Proposition 3. The result also follows from weak clustering if the asymptotic state $\phi_{\infty}$ is permutation invariant. Indeed, we could then average first over the time $t_{k_{p}}$ that appears only once and use weak clustering to conclude that this average is zero. According to Proposition 2, this occurs when $(\mathfrak{A}, \Theta, \phi)$ is strongly clustering, hence weakly clustering and $\phi_{\infty}$ automatically permutation invariant.

b. Condition (20) is implied by strong clustering (6.a) because of the equivalence of (6.a) and (6.b) and implies weak clustering (5). In fact, with $\widetilde{Y}:=Y-\phi(Y)$, condition (20) 
means that

$$
\lim _{t \rightarrow \infty} \phi(X \tilde{Y}(t) Z)=\lim _{t \rightarrow \infty} \phi(X(-t) \tilde{Y} Z(-t))=0
$$

As far as fluctuations are concerned, permutation invariance (16) implies

Corollary 1. Let the dynamical system $(\mathfrak{A}, \Theta, \phi)$ be weakly clustering. Let the multiple average (14) exist and define an asymptotic state $\phi_{\infty}$ which is permutation invariant in the sense of (16). Then, with $X^{(1)}, \ldots, X^{(r)}$ centred observables of $\mathfrak{A}$,

$\lim _{N \rightarrow \infty} \phi_{\infty}\left(F_{N}\left(X^{(1)}\right) F_{N}\left(X^{(2)}\right) \cdots F_{N}\left(X^{(r)}\right)\right)= \begin{cases}0 & r=2 n+1 \\ \sum_{\nu, \text { ord }}^{(2)} \phi_{\infty}\left(X_{\nu(1)}^{(1)} \cdots X_{\nu(2 n)}^{(2 n)}\right) & r=2 n\end{cases}$

where $\sum_{\nu \text {, ord }}{ }^{(2)}$ means that the sum is over all ordered pair partitions $\nu=\left(\left(\alpha_{1}, \beta_{1}\right)\right.$, $\left.\left(\alpha_{2}, \beta_{2}\right), \ldots,\left(\alpha_{n}, \beta_{n}\right)\right)$ of $\{1,2, \ldots, 2 n\}$, i.e. we choose sites $\alpha_{j}<\beta_{j}$ such that $\nu\left(\alpha_{j}\right)=$ $\nu\left(\beta_{j}\right)=j$ with $j$ running from 1 to $n$ and $\alpha_{1}<\alpha_{2}<\cdots<\alpha_{n}$.

Proof: An easy consequence of Proposition 3.

Corollary 2. If the dynamical system $(\mathfrak{A}, \Theta, \phi)$ is strongly clustering, then the fluctuations $F_{N}(X)$ of observables $X=X^{*} \in \mathfrak{A}$ such that $\phi(X)=0$ and $\phi\left(X^{2}\right)=\sigma^{2}$ tend to Gaussian random variables with zero mean and variance $\sigma$.

Proof: Since $\phi(X)=0$ implies $\phi_{\infty}\left(X_{j}\right)=0$, for all $j \in \mathbf{N}_{0}$, we are in fact dealing with centred words in $\mathfrak{A}_{\infty}$. Because of Proposition 2, we can apply Corollary 1, using the notation for pair partitions introduced there, to compute the even moments (the odd ones are zero)

$$
M_{2 n}:=\lim _{N} \phi_{\infty}\left(F_{N}(X)^{2 n}\right)
$$

As in Proposition 2, it follows that

$$
\phi_{\infty}\left(X_{\nu(1)} X_{\nu(2)} \cdots X_{\nu(2 n)}\right)=\prod_{j=1}^{n} \phi_{\infty}\left(X_{\nu\left(\alpha_{j}\right)} X_{\nu\left(\beta_{j}\right)}\right)=\sigma^{2 n}
$$

where we have used that $\phi_{\infty}\left(X_{\nu\left(\alpha_{j}\right)} X_{\nu\left(\beta_{j}\right)}\right)=\phi_{\infty}\left(\left(X^{2}\right)_{j}\right)=\phi\left(X^{2}\right)=\sigma^{2}$. Since the number of pair partitions $\nu:\{1,2, \ldots, 2 n\} \mapsto\{1,2, \ldots, n\}$ is $\prod_{j=0}^{n}\left(\begin{array}{c}2(n-j) \\ 2\end{array}\right)=(2 n) ! / 2^{n}$, we get

$$
M_{2 n}=(2 n-1) ! ! \sigma^{2 n},
$$


that is the $2 n$-th moment of the Gaussian distribution $g_{0, \sigma}(t)$ with zero mean and variance $\sigma: g_{0, \sigma}(t)=1 / \sqrt{2 \pi \sigma^{2}} \exp \left(-t^{2} / 2 \sigma^{2}\right)$.

Proposition 3 is sufficient to guarantee that the linear functional $\Phi$ defined as a pointwise limit by $(19)$ is positive on the free $*$-algebra $\mathcal{F}$ generated by all possible fluctuations $F(X), X \in \mathfrak{A}$, where $F(X)^{*}:=F\left(X^{*}\right)$. Furthermore, one can look for concrete Hilbert space representations of the abstract couples $(\mathcal{F}, \Phi)$. More precisely, one may search for a structure determined by creation and annihilation operators $a^{*}$ and $a$ satisfying certain commutation relations [14] and for a "ground" state $\Omega$ such that

$$
\left\langle\Omega,\left(a+a^{*}\right)^{n} \Omega\right\rangle=\Phi\left(F(X)^{n}\right) .
$$

It is, however, clear that associating definite probability distributions and representations to fluctuations very much depends on how well-behaved the even moments in Proposition 3 are. There are a few cases, Corollary 2 being one of them, where a definite structure emerges. We present two of them, but first recall the notion of non-crossing pair partitions.

Let $\nu$ be a pair partition of $\{1,2, \ldots, 2 n\}$ into $n$ pairs $\left(\alpha_{j}, \beta_{j}\right)$, with $\alpha_{j}<\beta_{j}$, such that $\nu\left(\alpha_{j}\right)=\nu\left(\beta_{j}\right)=j, j \in\{1,2, \ldots, n\}$. A crossing occurs when

$$
\alpha_{j}<\alpha_{k}<\beta_{j}<\beta_{k} \quad \text { for some } j, k \in\{1,2, \ldots, n\} .
$$

Denoting by $c(\nu)$ the number of crossings in a pair partition $\nu, \nu$ is non-crossing if $c(\nu)=0$. If $\nu$ is a non-crossing pair partition of $\{1,2, \ldots, 2 n\}$, then its pairs $\left(\alpha_{j}, \beta_{j}\right), j=1,2, \ldots, n$, are nested, that is if $\alpha_{j}<\alpha_{k}<\beta_{j}$ for some $j, k$, then also $\beta_{k}<\beta_{j}$.

- Bosonic Brownian motion

$$
\phi_{\infty}\left(X_{\nu(1)}^{(1)} \cdots X_{\nu(2 n)}^{(2 n)}\right)=\prod_{k=1}^{n} \phi\left(X^{\left(\alpha_{k}\right)} X^{\left(\beta_{k}\right)}\right) .
$$

can be represented in terms of Bosonic creation and annihilation operators, i.e. $a a^{*}-$ $a^{*} a=\mathbb{I}$.

- Free Brownian motion

$$
\phi_{\infty}\left(X_{\nu(1)}^{(1)} \cdots X_{\nu(2 n)}^{(2 n)}\right)=\delta_{0, c(\nu)} \prod_{k=1}^{n} \phi\left(X^{\left(\alpha_{k}\right)} X^{\left(\beta_{k}\right)}\right) .
$$

can be represented on the full Fock space in terms of creation and annihilation operators satisfying $a a^{*}=\mathbb{I}$.

In the case of the Bosonic Brownian motion, the ground state distribution is Gaussian. In the case of the Free Brownian motion the ground state distribution is the semicircle law and to it only non-crossing pair partitions contribute [7]. Indeed, the semicircle distribution

$$
\gamma_{0,1}(t)=\frac{1}{2 \pi} \sqrt{4-t^{2}}, \quad|t| \leq 2
$$


has vanishing odd moments, whereas the even ones are given by the Catalan numbers $C_{n}$ : $M_{2 n}=\left(\begin{array}{c}2 n \\ n\end{array}\right) /(n+1)=C_{n}$. The $C_{n}$ are defined by the recursion relation

$$
C_{0}=1, \quad C_{n}=\sum_{k=1}^{n} C_{k-1} C_{n-k}
$$

The Catalan numbers count in how many different ways one can partition $\{1,2, \ldots, 2 n\}$ in ordered non-crossing pairs $\left\{\left(\alpha_{1}, \beta_{1}\right),\left(\alpha_{2}, \beta_{2}\right), \ldots,\left(\alpha_{n}, \beta_{n}\right)\right\}$, with $\alpha_{1}<\alpha_{2}<\cdots<\alpha_{n}$.

In the following proposition, we exhibit conditions on the dynamical system $(\mathfrak{A}, \Theta, \phi)$ and on the procedure of averaging multi-time correlation functions such that a free statistics for time-asymptotic fluctuations arises. We start by observing that the explicit rule (14) for computing averages has a natural product structure: if $\mathbf{t} \mapsto f_{1}\left(t_{i_{1}}, t_{i_{2}}, \ldots, t_{i_{m}}\right)$ and $\mathbf{t} \mapsto f_{2}\left(t_{j_{1}}, t_{j_{2}}, \ldots, t_{j_{n}}\right)$ are uniform limits of multi-time correlation functions, then

$$
\left\{i_{1}, i_{2}, \ldots, i_{m}\right\} \cap\left\{j_{1}, j_{2}, \ldots, j_{n}\right\}=\emptyset \Longrightarrow \operatorname{Avg}\left(f_{1} f_{2}\right)=\operatorname{Avg}\left(f_{1}\right) \operatorname{Avg}\left(f_{2}\right)
$$

Notice, however, that an average is generally not specified if we impose the product property and specify all single-time averages Avg. Indeed, e.g. a bounded function $f$ over $\mathbf{Z}^{2}$ is not necessarily the uniform limit of linear combinations of products of bounded functions $t_{1}, t_{2} \mapsto h\left(t_{1}\right) g\left(t_{2}\right)$ over $\mathbf{Z}$.

Proposition 4. Let $(\mathfrak{A}, \Theta, \phi)$ satisfy condition (20) and let the multiple average (14) exist and define an asymptotic state $\phi_{\infty}$ on $\mathfrak{A}_{\infty}$. Let us also assume that for any time independent choice of observables $A$ and $C$ and centred observables $X, Y$ and $B$

$$
\operatorname{Avg}(t \mapsto \phi(A X(t) B Y(t) C))=0
$$

Then, if $X^{(1)}, \ldots, X^{(2 n)}$ are centred observables in $\mathfrak{A}$,

$$
\lim _{N \rightarrow \infty} \phi_{\infty}\left(F_{N}\left(X^{(1)}\right) F_{N}\left(X^{(2)}\right) \cdots F_{N}\left(X^{(2 n)}\right)\right)=\frac{1}{n !} \sum_{\nu}^{(2)} \delta_{0, c(\nu)} \prod_{k=1}^{n} \phi\left(X^{\left(\alpha_{k}\right)} X^{\left(\beta_{k}\right)}\right)
$$

where the sum extends over all pair partitions $\nu=\left(\left(\alpha_{1}, \beta_{1}\right),\left(\alpha_{2}, \beta_{2}\right), \ldots,\left(\alpha_{n}, \beta_{n}\right)\right)$ of $\{1,2, \ldots, 2 n\}$. If the asymptotic state $\phi_{\infty}$ is also permutation invariant, then

$$
\lim _{N \rightarrow \infty} \phi_{\infty}\left(F_{N}\left(X^{(1)}\right) F_{N}\left(X^{(2)}\right) \cdots F_{N}\left(X^{(2 n)}\right)\right)=\sum_{\nu, \text { ord }}^{(2)} \delta_{0, c(\nu)} \prod_{k=1}^{n} \phi\left(X^{\left(\alpha_{k}\right)} X^{\left(\beta_{k}\right)}\right)
$$

where the sum now extends over all ordered pair partitions $\nu$ of $\{1,2, \ldots, 2 n\}$.

Proof: As the asymptotic state $\phi_{\infty}$ is defined according to (14), we know it to be strongly compatible and endowed with the product structure (23). It need not be, however, permutation invariant. In any case, on the basis of Proposition 3, we only have to consider 
even moments of order $2 n$. More precisely, we can restrict to expectations of the form $\phi_{\infty}\left(X_{\nu(1)}^{(1)} \cdots X_{\nu(2 n)}^{(2 n)}\right)$, where $\nu$ is a pair partition of $\{1,2, \ldots, 2 n\}$. In order to compute such an expectation, we must perform successive time-averages as in (14). We begin with averaging with respect to $t_{1}$

$$
\operatorname{Avg}\left(t_{1} \mapsto \phi\left(X^{(1)}\left(t_{\nu(1)}\right) X^{(2)}\left(t_{\nu(2)}\right) \cdots X^{(2 n)}\left(t_{\nu(2 n)}\right)\right)\right)
$$

Either the time $t_{1}$ appears in two consecutive words $X^{(j)}$ and $X^{(j+1)}$, in which case we can use weak clustering to obtain

$$
\begin{aligned}
& \operatorname{Avg}\left(t_{1} \mapsto \phi\left(X^{(1)}\left(t_{\nu(1)}\right) X^{(2)}\left(t_{\nu(2)}\right) \cdots X^{(2 n)}\left(t_{\nu(2 n)}\right)\right)\right) \\
& \left.\quad=\phi\left(X^{(j)} X^{(j+1)}\right) \phi\left(X^{(1)}\left(t_{\nu(1)}\right) \cdots X^{(j-1)}\left(t_{\nu(j-1)}\right) X^{(j+2)}\left(t_{\nu(j+2)}\right) \cdots X^{(2 n)}\left(t_{\nu(2 n)}\right)\right)\right) .
\end{aligned}
$$

Or, the two words at time $t_{1}$ are separated by one or more other words at different times: $\phi\left(w X^{\left(\alpha_{1}\right)}\left(t_{1}\right) w^{\prime} X^{\left(\beta_{1}\right)}\left(t_{1}\right) w^{\prime \prime}\right)$. In this case we use condition $(24)$ as follows. Notice that $w^{\prime}$ is not necessarily centred and so we write

$$
w^{\prime}=\left(w^{\prime}-\phi\left(w^{\prime}\right) \mathbb{I}\right)+\phi\left(w^{\prime}\right) \mathbb{I} .
$$

Then, remembering that the time $t_{1}$ does not appear in $w, w^{\prime}, w^{\prime \prime}$, condition (24) and weak clustering imply

$$
\begin{aligned}
& \operatorname{Avg}\left(t_{1} \mapsto \phi\left(w X^{\left(\alpha_{1}\right)}\left(t_{1}\right) w^{\prime} X^{\left(\beta_{1}\right)}\left(t_{1}\right) w^{\prime \prime}\right)\right) \\
& =\phi\left(w^{\prime}\right) \operatorname{Avg}\left(t_{1} \mapsto \phi\left(w\left(X^{\left(\alpha_{1}\right)} X^{\left(\beta_{1}\right)}\right)\left(t_{1}\right) w^{\prime \prime}\right)\right) \\
& =\phi\left(X^{\left(\alpha_{1}\right)} X^{\left(\beta_{1}\right)}\right) \phi\left(w^{\prime}\right) \phi\left(w w^{\prime \prime}\right) .
\end{aligned}
$$

Consider now the average with respect to $t_{2}$. This is either a similar average as that with respect to $t_{1}$ or else the time $t_{2}$ appears separately in each of the factors $\phi\left(w^{\prime}\right)$ and $\phi\left(w w^{\prime \prime}\right)$ in (25). If so, the average with respect to $t_{2}$ vanishes because of weak clustering and the centredness of the $X$ 's. Repeating this argument for all the time averages, we see that only nested pairs i.e. non-crossing pair partitions, contribute whence the result.

Proposition 4 and permutation invariance of the asymptotic state provide us with sufficient conditions on the correlation functions to yield fluctuations which are free random variables and hence semicircularly distributed.

Corollary 3. Let the dynamical system $(\mathfrak{A}, \Theta, \phi)$ satisfy conditions (20) and (24) and the multiple average (14) exist and define a permutation invariant asymptotic state $\phi_{\infty}$ as in (16). Then, the fluctuations $F_{N}(X)$ of observables $X=X^{*} \in \mathfrak{A}$ such that $\phi(X)=0$ 
and $\phi\left(X^{2}\right)=\sigma^{2}$ tend to semicircularly distributed random variables with zero mean and variance $\sigma$.

Proof: We can apply the previous proposition to compute the even moments (the odd ones are zero)

$$
M_{2 n}:=\lim _{N} \phi_{\infty}\left(F_{N}(X)^{2 n}\right)=C_{n} \sigma^{2 n},
$$

where $C_{n}$ are the Catalan numbers $(22)$. On the other hand, the latter moments are the non-zero moments of the semicircle distribution $\gamma_{0, \sigma}(t)=\sqrt{4 \sigma^{2}-t^{2}} / 2 \pi \sigma^{2}$ on $|t| \leq 2 \sigma$.

\section{Remarks}

a. Referring to the types of clustering we presented in Section 2, clustering in the mean (4) would not be sufficient to yield the result of Proposition 4, while strong clustering (6.a) is incompatible with condition (24) and with freeness, as already observed in Section 3. In the proof of Proposition 4, weak clustering (5) has been used in an essential way. Indeed, suppose that we assume instead of weak clustering only clustering in the mean. In the process of centring the observable $w^{\prime}$, sandwiched between two equal time observables $X^{\left(i_{k}\right)}\left(t_{k}\right)$ and $X^{\left(j_{k}\right)}\left(t_{k}\right)$, some other time $t_{\ell}$ might appear once in both $w^{\prime}$ and in $w w^{\prime \prime}$. Therefore, we should consider an average of a product $t \mapsto f(t) g(t)$ which is generally unequal to the product of the averages

$$
\operatorname{Avg}(t \mapsto f(t) g(t)) \neq \operatorname{Avg}(t \mapsto f(t)) \operatorname{Avg}(t \mapsto g(t))
$$

b. Conditions (20) and (24) are independent. Obviously, the latter does not imply the first one. As a counterexample to $(20) \Rightarrow(24)$, consider the algebra $\mathfrak{A}$ generated by linear combinations of the unitary operators $W(\mathbf{p}), \mathbf{p}=\left(p_{1}, p_{2}\right) \in \mathbf{Z}^{2}$, such that

$$
W(\mathbf{p})^{*}=W(-\mathbf{p}), \quad W(\mathbf{p}) W(\mathbf{q})=\mathrm{e}^{i \pi \theta \sigma(\mathbf{p}, \mathbf{q})} W(\mathbf{p}+\mathbf{q}),
$$

where $\theta \in[0,1)$ and $\sigma(\mathbf{p}, \mathbf{q})=p_{1} q_{2}-p_{2} q_{1}$. By equipping $\mathfrak{A}$ with the tracial state $\phi(W(\mathbf{p}))=\delta_{\mathbf{p}, \mathbf{0}}$ and the automorphisms $\Theta^{t}\left(W(\mathbf{p})=W\left(T^{t} \mathbf{p}\right)\right.$, where $T$ is the typical $2 \times 2$ matrix of the hyperbolic dynamics on the two-dimensional torus, one obtains the class of quantized cat-maps studied in [15]. A thorough examination of such models in the framework presently developed will be the subject of a forthcoming paper [16]. Here, it is sufficient to mention that there are values of $\theta$ for which $\lim _{t} \theta \sigma\left(T^{t} \mathbf{p}, \mathbf{q}\right) \bmod 1=\beta \Delta(\mathbf{p}, \mathbf{q})$ with $\beta$ and $\Delta(\mathbf{p}, \mathbf{q})$ two not necessarily zero quantities.

One calculates $\phi\left(W\left(T^{t_{1}} \mathbf{p}\right) W\left(T^{t_{2}} \mathbf{q}\right) W(-\mathbf{p}) W\left(-T^{t_{2}} \mathbf{q}\right)\right)=\mathrm{e}^{2 \pi i \theta \sigma\left(T^{t_{2}} \mathbf{q}, \mathbf{p}\right)} \delta_{T^{t_{1}} \mathbf{p}, \mathbf{p}}$. Thus, $\operatorname{Avg}\left(t \mapsto \phi\left(W(\mathbf{p}) W\left(T^{t} \mathbf{q}\right) W(-\mathbf{p}) W\left(-T^{t} \mathbf{q}\right)\right)\right)=\mathrm{e}^{2 \pi i \beta \Delta(\mathbf{q}, \mathbf{p})}$, whereas

$\lim _{t_{1}, t_{2} \rightarrow \infty} \phi\left(W\left(T^{t_{1}} \mathbf{p}\right) W\left(T^{t_{2}} \mathbf{q}\right) W(-\mathbf{p}) W\left(-T^{t_{2}} \mathbf{q}\right)\right)=0$. 


\section{Examples}

We implement in this section the constructions of above for a few simple model systems. More complex dynamical systems, with richer behaviour of correlation functions, will be considered in a forthcoming paper [16]. As Proposition 2 and Corollary 1 deal with the asymptotic state and the fluctuations of strongly clustering dynamical systems, we shall only consider here more extremely non-commuting dynamical systems.

As a first model, we consider a probability space $(X, \mu)$ equipped with an automorphism $\theta$ i.e. a measurable transformation $\theta$ of $X$, with measurable inverse $\theta^{-1}$, such that $\mu=\mu \circ \theta=\mu \circ \theta^{-1}$. We will assume that $\theta$ is mixing

$$
\lim _{n \rightarrow \infty} \mu\left(f g \circ \theta^{n}\right)=\mu(f) \mu(g) \quad \text { for all } \quad f, g \in \mathcal{L}^{1}(X, \mu)
$$

In the Koopman description of the classical dynamical system $(X, \mu, \theta)$, one considers the Hilbert space $\mathfrak{H}:=\mathcal{L}^{2}(X, \mu)$ and the unitary single step evolution $U f:=f \circ \theta$ of the "wave functions" $f$. The expectation $\mu(f)$ of an $\mathcal{L}^{\infty}$ function on $X$ is recovered as

$$
\mu(f)=\left\langle\mathbf{1}, M_{f} \mathbf{1}\right\rangle
$$

In this formula 1 denotes the constant function 1 on $X$ and $M_{f}$ the multiplication operator on $\mathfrak{H}$ by the function $f$. We now consider a "non-canonical" quantization whereby the quantum evolution of "wave-functions" is exactly the classical one [17]. In such a description, one may choose for $\mathfrak{A}$ the algebra of compact operators $\mathbf{C} \mathbb{I}+\mathfrak{K}(\mathfrak{H})$ to which a unit has been added. The dynamics is determined by the usual Heisenberg picture: $\Theta(X):=U X U^{*}$ and the reference state $\phi$ is the vector state defined by $\mathbf{1}$. Using the assumed mixing property of $(X, \mu, \theta)$, one readily verifies that whenever $X \in \mathfrak{K}(\mathfrak{H})$ and $w$ and $w^{\prime}$ are products in $\mathfrak{A}_{\infty}$ of copies of compact operators, such that both the rightmost letter of $w$ and the leftmost of $w^{\prime}$ are different from $j$, then

$$
\phi_{\infty}\left(w X_{j} w^{\prime}\right)=\phi(X) \phi_{\infty}(w) \phi_{\infty}\left(w^{\prime}\right)
$$

even when $w$ or $w^{\prime}$ contain letters pertaining to the index $j$. As a consequence,

$$
\phi_{\infty}\left(X_{\nu(1)}^{(1)} X_{\nu(2)}^{(2)} \cdots X_{\nu(n)}^{(n)}\right)=\prod_{j=1}^{n} \phi\left(X^{(j)}\right)
$$

whenever $X^{(j)} \in \mathfrak{K}(\mathfrak{H})$ and $\phi_{\infty}$ is permutation invariant. For a compact $X \in \mathfrak{K}(\mathfrak{H})$ we will denote by $\tilde{X}$ the element $X-\phi(X) \mathbb{I}$ obtained by centring $X$. In spite of good ergodic properties of $(X, \theta, \mu)$, its "quantization" $(\mathfrak{A}, \Theta, \phi)$ is not weakly clustering as can be seen from

$$
\lim _{t \rightarrow \infty} \phi\left(X \Theta^{t}(Y) X\right)=\phi(X)^{2} \phi(Y) \neq \phi\left(X^{2}\right) \phi(Y)
$$


$X$ and $Y$ compact. Considering fluctuations is not meaningful in such a case. In fact, expectations of third order moments of fluctuations $F_{N}(X)$ diverge with $N$ as can be seen from

$$
\phi_{\infty}\left(F_{N}(X)\right)=\frac{1}{\sqrt{N}} \phi\left(\tilde{X}^{3}\right)-\frac{N-1}{\sqrt{N}} \phi(X)\left(\phi\left(X^{2}\right)-\phi(X)^{2}\right)
$$

with $\tilde{X}:=X-\phi(X) \mathbb{I}$.

As a second example, we consider a Fermionic dynamical system whose observables are given by a CAR-algebra $\mathfrak{A}(\mathfrak{H})$ over a single-particle space $\mathfrak{H} \cdot \mathfrak{A}(\mathfrak{H})$ is generated by an identity $\mathbb{I}$ and by creation and annihilation fields $\left\{a^{*}(\varphi) \mid \varphi \in \mathfrak{H}\right\}$ and $\{a(\varphi) \mid \varphi \in \mathfrak{H}\}$ subject to the relations:

$$
\begin{aligned}
& \varphi \mapsto a^{*}(\varphi) \text { is C-linear and } \\
& \{a(\varphi), a(\psi)\}=0 \quad \text { and } \quad\left\{a(\varphi), a^{*}(\psi)\right\}=\langle\varphi, \psi\rangle .
\end{aligned}
$$

The parity automorphism $\pi$ on $\mathfrak{A}(\mathfrak{H})$ is determined by $\pi\left(a^{*}(\varphi)\right):=-a^{*}(\varphi)$ and a $*-$ automorphism $\Theta$ is said to be even if $\Theta \circ \pi=\pi \circ \Theta$. We assume furthermore that the dynamics determined by such an even $\Theta$ is asymptotically "Abelian" in the sense that

$$
\lim _{n \rightarrow \infty} \|\left\{a^{*}(\varphi), \Theta\left(\left(a^{\#}(\psi)\right)\right\} \|=0,\right.
$$

where $a^{\#}$ denotes either $a$ or $a^{*}$. It is well-known that a reference state $\phi$, invariant under an even, asymptotically Abelian $\Theta$, is automatically even i.e. $\phi \circ \pi=\phi$. Finally, we shall assume that the dynamical system is multi-clustering

$$
\lim _{t_{j}-t_{j+1} \rightarrow \infty} \phi\left(X^{(1)}\left(t_{1}\right) X^{(2)}\left(t_{2}\right) \cdots X^{(k)}\left(t_{k}\right)\right)=\prod_{j=1}^{k} \phi\left(X^{(j)}\right)
$$

with $t_{1}>t_{2}>\cdots>t_{k}$. The asymptotics of multi-correlation functions can be computed along the same lines as that for the strongly clustering case and we obtain when each of the $X^{(j)}$ is either even or odd

$$
\phi_{\infty}\left(X_{\nu(1)}^{(1)} X_{\nu(2)}^{(2)} \cdots X_{\nu(n)}^{(n)}\right)=\epsilon \prod_{p=1}^{k} \phi_{\infty}\left(\vec{\prod}_{i \in I_{p}} X^{(i)}\right) .
$$

$I_{p}$ is, as in Proposition 2, the set of all indices $j$ such that $\nu(j)=p$ and $\epsilon$ is either 1 or -1 according to whether an even or odd permutation is needed to permute the odd $X^{(j)}$ appearing in $X_{\nu(1)}^{(1)} X_{\nu(2)}^{(2)} \cdots X_{\nu(n)}^{(n)}$ into the order in which they appear in the product at the right-hand side of (27). This amounts to saying that $\phi_{\infty}$ is the Chevalley product of a countable number of copies of $\phi$ on the twisted tensor product $\mathfrak{A}\left(\oplus^{\mathbf{N}_{0}} \mathfrak{H}\right)$ of $\mathbf{N}_{0}$ copies of $\mathfrak{A}(\mathfrak{H})$ with itself. The fluctuations of creation and annihilation fields are now straightforwardly computed, yielding

$$
\lim _{N \rightarrow \infty} \phi_{\infty}\left(F_{N}\left(a^{\#}\left(\varphi_{1}\right)\right) F_{N}\left(a^{\#}\left(\varphi_{2}\right)\right) \cdots F_{N}\left(a^{\#}\left(\varphi_{n}\right)\right)\right)=\phi_{\mathrm{QF}}\left(a^{\#}\left(\varphi_{1}\right) a^{\#}\left(\varphi_{2}\right) \cdots a^{\#}\left(\varphi_{n}\right)\right) .
$$


$\phi_{\mathrm{QF}}$ is the "quasi-free projection" of the state $\phi$, namely the quasi-free state on $\mathfrak{A}(\mathfrak{H})$ determined by the covariance

$$
(\varphi, \psi) \mapsto \phi\left(a^{\#}(\varphi) a^{\#}(\psi)\right)
$$

We recover hereby the Fermionic central limit theorem of [18].

In its most basic form, the "free shift" is a quantum shift

$$
\Theta\left(e_{i}\right):=e_{i+1}, \quad i \in \mathbf{Z}
$$

on a set of generators $\left\{e_{i} \mid i \in \mathbf{Z}\right\}$ that satisfy the relations

$$
e_{i}^{*}=e_{i} \quad \text { and } \quad e_{i}^{2}=\mathbb{I}, \quad i \in \mathbf{Z} .
$$

The algebra $\mathfrak{A}$ of observables is the universal $\mathrm{C}^{*}$-algebra generated by $\mathbb{I}$ and the $e_{i}$. The finite linear combinations of monomials of the type $e_{i_{1}} e_{i_{2}} \cdots e_{i_{m}}$ with $i_{k} \neq i_{k+1}$ form a dense *-subalgebra $\mathfrak{A}_{0}$ of $\mathfrak{A}$. The product of two monomials determined by ordered index sets $\left\{i_{1}, i_{2}, \ldots, i_{m}\right\}$ and $\left\{j_{1}, j_{2}, \ldots, j_{n}\right\}$ is the monomial corresponding to the index set obtained by first concatenating $\left\{i_{1}, i_{2}, \ldots, i_{m}\right\}$ and $\left\{j_{1}, j_{2}, \ldots, j_{n}\right\}$ and then omitting those indices that appear twice in subsequent positions. The identity corresponds to the monomial with empty index set and the adjoint of a monomial is the monomial with reversed index set. If there are no preferred observables to single out apart from the identity, a meaningful reference state is the state $\phi$ on $\mathfrak{A}$

$$
\phi\left(e_{i_{1}} e_{i_{2}} \cdots e_{i_{m}}\right)=0 \text { if } m>0 \quad \text { and } \quad \phi(\mathbb{I})=1
$$

The dynamical system $(\mathfrak{A}, \Theta, \phi)$ is weakly, but not strongly, clustering. It is quite straightforward to compute the asymptotic state $\phi_{\infty}$ : it is the free product $*_{i \in \mathbf{N}_{0}} \phi$ of copies of $\phi$. As each centred element in $\mathfrak{A}_{0}$ is a finite linear combination of monomials with non-trivial dependence set, it suffices to show that for $n=1,2, \ldots$ the expectation of an element $X_{\nu(1)}^{(1)} X_{\nu(2)}^{(2)} \cdots X_{\nu(n)}^{(n)}$ in the state $\phi_{\infty}$ vanishes, where each $X^{(k)}$ is a non-trivial monomial

and consecutive $\nu(k)$ are different. Clearly, when all differences $\left|t_{k}-t_{\ell}\right|$ for $k \neq \ell$ appearing in the list of $\nu(j)$ 's become sufficiently large, there is no possible simplification in the monomial $X^{(1)}\left(t_{\nu(1)}\right) X^{(2)}\left(t_{\nu(2)}\right) \cdots X^{(n)}\left(t_{\nu(n)}\right)$ due to the rule $e_{i}^{2}=\mathbb{I}$ and because of $(28)$ $\phi\left(X^{(1)}\left(t_{\nu(1)}\right) X^{(2)}\left(t_{\nu(2)}\right) \cdots X^{(n)}\left(t_{\nu(n)}\right)\right)=0$ and so $\phi_{\infty}\left(X_{\nu(1)}^{(1)} X_{\nu(2)}^{(2)} \cdots X_{\nu(n)}^{(n)}\right)=0$ too. The state $\phi_{\infty}$ is permutation invariant and satisfies even a strengthened Condition (24), where the average is replaced by a limit. Temporal fluctuations of centred self-adjoint observables are therefore, by Proposition 4, semicircularly distributed.

\section{Acknowledgements:}

F.B. acknowledges financial support from the Onderzoeksfonds K.U.Leuven F/97/60 and the Italian I.N.F.N. and M. De Cock acknowledges financial support from FWO-project G.0239.96. 


\section{References}

[1] Casati G., Chirikov B.V., Izrailev F.M., Ford J.: Stochastic behaviour of a quantum pendulum under a periodic perturbation, Lecture Notes in Physics 93, 334-352, New York: Springer Verlag, 1979

[2] Haake F., Kus M., Scharf R.: Classical and quantum chaos for a kicked top. Z. Phys. B65, 381-395 (1987)

[3] Berry M.V., Balazs N.L., Tabor M., Voros E.: Quantum maps. Ann. Phys. 122, 26-63 (1979)

[4] Balazs N.L., Voros A.: The quantized baker's transformation. Ann. Phys. 190, 1-31 (1989)

[5] Casati G., Chirikov B.V.: Quantum Chaos. Cambridge: Cambridge University Press, 1995

[6] Benatti F., Fannes M.: Statistics and quantum chaos, J. Phys. A, in press

[7] Speicher R.: Generalized statistics of macroscopic fields. Lett. Math. Phys. 27, 97-104 (1993)

[8] Voiculescu D.V., Dykema K.J., Nica A.: Free Random Variables. Providence,RI: AMS 1992

[9] Emch G.G.: Algebraic Methods in Statistical Mechanics and Quantum Field Theory. New York: Wiley, 1974

[10] Narnhofer H., Thirring W.: Mixing properties of quantum systems. J. Stat. Phys. 57, 811-825 (1989)

[11] Greenleaf F.P.: Invariant Means on Topological Groups. New York: Van Nostrand Reinhold, 1969

[12] Goderis D., Verbeure A., Vets P.: Non-commutative central limits. Probab. Th. Rel. Fields 82, 527-544 (1989)

[13] Speicher R., Waldenfels W. von: A general central limit theorem and invariance principle, Quantum Probability and Related Topics IX, 371-387 Singapore: World Scientific, 1994 
[14] van Leeuwen H., Maassen H.: A q-deformation of the Gauss distribution. J. Math. Phys. 36, 4743-4756 (1996)

[15] Benatti F., Narnhofer H., Sewell G.L.: A non-commutative version of the Arnold cat map. Lett. Math. Phys. 21, 157-192 (1991)

[16] Andries J., Benatti F., De Cock M. and Fannes M.: Dynamical fluctuations in quantized toral automorphisms, in preparation

[17] Berry M.: True quantum chaos? An instructive example, Proceedings of the Yukawa symposium, Tokyo (1990)

[18] Hudson R.L.: A quantum mechanical central limit theorem for anti-commuting observables. J. Appl. Prob. 10, 502-509 (1973) 